\title{
Applications of particle accelerators in medical physics.
}

\section{Giacomo Cuttone ${ }^{1}$}

INFN-Laboratori Nazionali del Sud

V. S. Sofia 44, 95123 Catania (Italy)

E-mail: Cuttone@lns.infn.it

Within an hadrontherapy center the particle accelerator is the key element; it is the center of operation clinical and it defines unequivocally the overall technological performance because it has an impact on the quality of the radiation treatments produced. For this reason, even if the machine represents a low percentage, not more than $20 \%$, of the total investment necessary for the implementation of a hadrontherapy centre, the choice is still the most qualifying for the Centre and on this chose is focused a lot of attention and the technological typical planning of these facilities. A great debate scientific, technological and not clinical at least, is furthered for decades on the choice between cyclotrons and synchrotrons, in light of the strengths and weaknesses of each of those two solutions. The physical characteristics, technological and clinical impact on the facilities from these two types of accelerators will be widely reported, also by the description of the choices already made in the main centers of hadrontherapy in operation or in advanced phase of realization in Europe and in the rest of the world. The future of the possible innovations (FFAG, Linac booster) also will be considered by placing attention to the new horizons offered by research in the field of the accelerators for protons and light ions.

XLVIII International Winter Meeting on Nuclear Physics - BORMIO2010

Bormio, Italy

January 25-29 2010

$1 \quad$ Speaker 


\section{Introduction}

Every year in the world, a growing number of patients are suffering from cancer and the request for adequate treatment and possibly free from suffering is in remarkable growth. Between the different possible methods to heal tumours, radiotherapy with charged particles is the more recently developed and that is in the form more attractive as regards the maintenance of a high quality of life. Up to a few years ago, with the exception of the two centres of Himac in Japan and Loma Linda in the USA, it was possible to use this technique exclusively within the research laboratories that had the accelerators able to accelerate protons and ions to energy appropriate to allow these therapies.

Only in the past decade with changing technology of the accelerators, automatic checks and of treatment protocols largely occurred, some companies such as the IBA, Siemens, Hitachi, Mitsubishi and ACCEL have begun to realize and to provide treatment plants complete for hadrontherapy. These treatment plants installed in hospital centres formally independent from research laboratories are generally composed of 4 components; the accelerator for the protons/ions, the systems of transport of the beam, the accessory equipment for the treatment of patients in the halls of treatment and control software and treatment. This new scenario has stimulated interest to the implementation of centres for hadrontherapy in many countries, Italy included. Moreover the use of accelerators for radioisiotope production to be used in advanced molecular imaging will be presented.

\section{Accelerators for Radioisotope production}

Both Radioisotopes and enriched stable isotopes are essential to a wide variety of applications in medicine, where they are used in the diagnosis and treatment of illness. The radioisotopes produced for medical applications are tipically used in nuclear medicine for diagnosis providing dynamic and functional information to study the organ functions. The labelled biomolecules and radioisotopes involved should have as ideal features the absence of beta particle emission, a half-life as short as possible and gamma energy emission in a range between 100 and $300 \mathrm{keV}$. For a long period the production of radioactive isotopes for medical applications was mainly based on neutron induced nuclear reactions. This was essentially done in nuclear reactors but their availability is slowly decreasing so that the accelerators based production facility are growing up. It is well known and proven that Short life radioisotopes $\left(\mathrm{F}^{18}, \mathrm{C}^{11}, \mathrm{~N}^{13}\right.$ and $\left.\mathrm{O}^{15}\right)$ are tipically accelerator produced radionuclides. Also others Radionuclides $\left(\mathrm{Ga}^{67}, \mathrm{In}^{111}, \mathrm{I}^{123}, \mathrm{Ta}^{201}\right)$ are produced by means of $30 \mathrm{MeV}$ cyclotrons for locoregional distribution. According to those a wide market of turn-key accelerators is nowadays available. The main choice in this field is represented by cyclotrons. Infact looking to the time structure of the extracted beam, cyclotrons represent the golden standard with respect to linacs. It si possible to state that the power deposition in a production target is significantly better using a cyclotron which it is characterized by a beam time structure that can be assumed as a " practically continuous beam". Linacs are characterized by a low repetition rate so that the instantaneous power deposition in the target so high that force to implement very complex solution expecially in the material choice for thermal dissipation. Modern isotope production facilities consists of a compact $\mathrm{H}^{-}$cyclotron in the energy range between 10 and $30 \mathrm{MeV}$ with 
extracted current up to $400 \mu \mathrm{A}$ and highly sophisticated target technology and chemistry. Target can be made solid, gas or liquid. In any case they have to be designed in order to dissipate up to $10 \mathrm{~kW}$. The choice of material is dependent on the particular nuclide process. Although a general rule does not exist, there are some aspects of target body like activation, contamination, corrosion and cooling, have to be kindly considered. Target can be either internal and external, placed at the end of dedicated beam transport lines. Different commercial solutions are available. IBA, General Electric, CTI, EBCO and SUMITOMO are the main cyclotron producers. They also offer dedicated radiochemistry modules for the production of the labelled molecula to be used.

\section{Beam featurs for a hadrontherapy center.}

The use of hadrons, particularly protons, in radiotherapy is a major innovation in the fight against cancer. However, the level of the definitions and specifications of a hadron beam is not yet well defined as in the case of other more conventional radioactive therapies. In this regard we should introduce the following definitions and specifications in accord with our experience and the data available in literature. Increased selectivity ballistics of hadrons, compared with photons and electrons used in conventional radiation therapy, requires compliance with stringent specifications on the distribution of dose in order to achieve a 3D conformal therapy.

The general characteristics that each hadrontherapy center must have are:

- ability to treat most cancers at any depth;

- treatment times as short as possible;

- high ability to discriminate between the volume to be treated and the healthy tissue that surrounds it and high dose uniformity in volume treated;

-availability of treatment rooms with access for beam fixed and mobile lines;

- great accuracy $(\leq 3 \%)$ in absolute and relative dosimetry;

- reliable control systems.

It is therefore necessary to define a beam of hadrons in terms of path, modulation of the Bragg peak, adjustment of the depth of penetration (range), dose rate, field size, uniformity and symmetry of the field, penumbra side, dose distal fall off.. The required characteristics for the beam hadrons used for the treatment have a decisive influence in the choice of accelerator to use. If one considers the dose curve as a function of depth, practical range defines the distance between the surface of the input beam of protons and distal point where the dose is reduced by $10 \%$. It depends, usually, on the energy and materials along the beam line (tools for monitoring beam, tools for adaptation range and beam modulation of the same). The range of a beam of 250 $\mathrm{MeV}$ protons is approximately $38 \mathrm{~cm}$ in water. The equivalent energy demanded to a carbon ion beam of 4800 is equal to $400 \mathrm{AMeV}$. The minimum range required for a line dedicated to the treatment of deep tumors is $22 \mathrm{~cm}$ in water. In the case of lines devoted to the treatment of not deep cancer as in the case of eye, treatments require energy protons between 60 and $70 \mathrm{MeV}$, whose range is $3-3.5 \mathrm{~cm}$ in water. The range of the beam could be amended by step of $0.2 \mathrm{~mm}$ in water for extensions less than $5 \mathrm{~cm}$ and by step of $1 \mathrm{~mm}$ for extensions more than $5 \mathrm{~cm}$. This can be achieved both by changing the energy of the beam and inserting absorber in front of the patient (Range Shifter). This solution has the greater inconvenience that it produces neutrons and light fragments (only in the case of ions) and the deterioration of the geometric 
characteristics of beam (emittance increase). So it can be used when the depth of treatment is no more than $45 \mathrm{~cm}$. Where it will require deeper treatments is preferable to insert absorber in strikeouts properly shielded and outside the treatment rooms where patients are located. This solution is always recommended in the case of using ions.

Regarding the Time Structure of beam it must show some considerations related to the scanning systems used and dosimeters used. In principle no one can have trouble in implementation of a system of passive diffusion. In designing a system of active scanning it must be placed particular care to a maximum fluctuation of intensity that you can check in a certain interval of time. On the other hand, the intensity of protons or ions per pulse, using synchrotron and linac, must be carefully assessed both in the design of the scanning system and beam characterization dosimetric. All these considerations led to choose the cyclotrons and synchrotron accelerators such election for external beam radiotherapy of hadrons.

\section{Cyclotrons and Synchrotrons: Advantages and Disadvantages}

From the beginning of its history in Berkley the cyclotron, has been used for applications in the medical field. Having generally the final energy beam accelerated fixed, it is appreciated from the point of view of the simplicity of its design and its ease of use. Moreover, the availability of superconductivity technology makes it feasible with reduced size and operating costs. The cyclotrons produce beams continue from the point of view of the structure and timing and with high intensity and stability. These characteristics make cyclotrons machines designed to perform an efficient system of active scanning. Moreover, because the acceleration process is extremely fast $(<20 \mu \mathrm{sec})$ it is possible to create fast ignition and shutdown systems. It is possible, therefore achieving fast electronic circuits for the stabilization of intensity beam with characteristic time of $100 \mathrm{msec}$. The high stability and constancy of beam extracted from a superconducting cyclotron, allows also greater governability in terms of electronic and less technical staff employed; From an economic point of view, this means a significant reduction of costs design, construction and operation of the machine.

The main disadvantage of cyclotrons is represented by the inability to continuously vary the energy of the extracted beam, so it needs using external systems based on modulation of passive absorber. These systems, moreover, completely decouple the accelerator from the patient. They are typically made of graphite and are far from the treatment room. The production of neutrons is great but easy to screen and the activation is limited given the current low beam used in hadrontherapy. These modulators are sufficiently fast to allow varying the range with steps of $5 \mathrm{~mm}$ in $100 \mathrm{msec}$. This time is lower than that of typical of a respiratory cycle $(2-4 \mathrm{sec})$. It is thus always possible to implement treatment techniques controlled by the cycle of the patient's respiration (breathing active treatment). The synchrotron are machines with a well-known technology producing pulsed beam with variable energy. By monitoring the number of cycles of acceleration is possible even varying energy of a few $\mathrm{MeV}$. The total weight of the machine is limited, but its dimensions are always greater than $10 \mathrm{~m}$ and $30 \mathrm{~m}$ in the case of machines for protons and ions. The beam is then extracted pulsed temporally and its intensity is limited and not constant. They are machines complex to use and require much attention and great experience to ensuring its efficient and reproducible operation. But the possibility to vary the energy beam extraction eliminates the need of systems of external 
modulation. The beam produced by a synchrotron also is extracted in discrete packages and typically it is present only for about half of the total time of exposure to the patient. For this reason, the scanning system must operate with speed double compared to that of a cyclotron. This implies the need to increase the power of the magnet system.

\section{Commercial Solutions for hadrontherapy centers}

A worldwide so far only five companies have already made at least one plant hadrontherapy complete, i.e. made up by the ladrons accelerators, as well as by transport systems, systems beam scanning, measurement systems and control of the dose and released facing radiation protection, access control and safety.

Feature common of these companies is that in addition to providing the complete system for hadrontherapy are also able to provide assistance project for the definition of technological systems and accessories. Below we will just give a quick overview of what made so far by these companies and the general characteristics of different types of plant of hadrotheray made by the various companies.

The IBA was the first private company to carry out a complete system of protontherapy at commercial level and able to treat hundreds of patients per year. The accelerator developed by the IBA is the Proteus 235 which is able to provide a beam of protons with an energy of 235 $\mathrm{MeV}$ and intensity of a beam extract of about $300 \mathrm{nA}$. This cyclotron, although not using superconductive technology, it is quite compact and power consumption are quite content thanks to the particular technical solutions adopted. This accelerator has been selected by 6 protonterapia centers around the world (one in Boston, one in Florida, one in Japan, one in Korea, two in China).

The IBA has developed a mechanical system for the insertion of various slices of calibrated thicknesses in order to make variable energy beam that in the case of cyclotrons is fixed. The change of system is supplemented by a energy magnetic picker that can provide a treatment room with beam at the desired energy and low energetic uncertainty. This system of variation is located near the cyclotron and therefore it allows to eliminate the modulator energy plants in protontherapy previous generation was positioned near the patient.

Recently, the IBA is also selling a superconducting cyclotrons, as a jointly project with INFN and JINR, capable to producing protons and ions beams at $400 \mathrm{AMeV}$. At present this the only cyclotron used for treatments with carbon ions. It has a diameter of 6 meters and a weight of 700 tonnes. Protons at $260 \mathrm{MeV}$ are extracted by stripping of the $\mathrm{H}^{+}$while carbon ions are extracted y electrostatic deflector placed in the valley.

The ACCEL company in 2001 started the construction of two superconducting cyclotrons able to provide $250-\mathrm{MeV}$ protons. These two cyclotrons have been ordered by the laboratory research of PSI Villigen (CH) and the protontherapy center Rinecker Munich (D), in particular this center has also applied for the supply of four Gantries. Also in collaboration with the PSI, ACCEL is implementing a new type of Gantry, more compact than the gantries produced by the IBA and best features from the point of view of the treatment.

The company ACCEL has developed its own system to select and change the energy of the beam extracted from the cyclotron. The cyclotron produced by ACCEL unlike that IBA is superconducting and therefore it has smaller size, about $2 \mathrm{~m}$. This cyclotron has been designed by a group of physicists of Michigan State University, leaders in the field of superconducting 
cyclotrons, and it has already designed and built the cyclotrons K-500 and K-1200 for the National Superconducting Cyclotron Laboratory (NSCL). Recently came into operation cyclotron at the clinic where PSI so far have already been treated about 30 patients.

The company HITACHI has extensive experience in building accelerators made for purposes of both scientific and medical research. Based on the experience gained since the'60s, HITACHI has developed a synchrotron for protons and ions aimed to therapy in the hospital. The company has supplied equipment for the "Proton Medical Research Center of Tsukuba and the" Wan Energy Research Center of Wakasa (Japan) is building the plant for protontherapy M.D. Anderson Cancer Center, Texas (USA).

The synchrotron of HITACHI provides a beam of protons with energy varying from 70 to $250 \mathrm{MeV}$ step with about $20 \mathrm{MeV}$, the circumference of the machine is approximately $23 \mathrm{~m}$ and the current intensity of $1.2510^{11}$ protons/pulse corresponding to about $10 \mathrm{nA}$. The frequency of repetition of the beam is approximately $0.5 \mathrm{~Hz}$.

The company MITSUBISHI thanks to a collaboration agreement with the center HIBMC of Hyogo (Japan) has released a set of accelerators for protontherapy and hadrontherapy and also in 2003 it inaugurated the center SHIZOUKA where it was installed a proton synchrotron. The synchrotron for $230 \mathrm{MeV}$ protons, installed at SHIZOUKA has characteristics similar to those reported for the HITACHI machine. Both HITACHI and MITSUBISHI synchrotrons are injected by a linac for protons.

The Siemens, through a collaboration agreement done with GSI, sold a synchrotron able to accelerate protons with energy between 48 and $220 \mathrm{MeV}$ and ion carbon beams at energy varying between 88 and $430 \mathrm{AMeV}$. The injection is through a linac at $7 \mathrm{AMeV}$ for all beams. The machine is capable of changing its energy in $1 \mathrm{sec}$. With a spread in energy of $0.2 \%$. It has a diameter of about $20 \mathrm{~m}$.

\section{Future Developments}

Research in the field of accelerator physics is surely also pushing development in the field of machinery applied to hadrontherapy. In this point of view an important position is represented by changes proposed by U. Amaldi and foundation TERA: Cyclinicas. The basic idea of the proposal is to couple with different cyclotrons the linacs to bring the final energy of the beam at that required for deep treatments. In the field of protontherapy the proposal is based on the idea to coupling cyclotrons for $30 \mathrm{MeV}$ protons to Linac of a $3 \mathrm{GHz}$ able to bring the final energy up to $250 \mathrm{MeV}$ (LIBO). More in general in hadrontherapy field was proposed to match a linear accelerator and the ion cyclotron INFN / IBA. The proposed Linac named CABOTO (CArbon BOoster for Therapy in Oncology) will consist of 16 modules at $3 \mathrm{GhZ}$ and it will have a total length of $22 \mathrm{~m}$. CABOTO will allow to bring the final ions energy from $300 \mathrm{AMeV}$ $435 \mathrm{AMeV}$ corresponding to a range in $31 \mathrm{~cm}$ of water. Both LIBO and CABOTO that are Linac type SCL (Side Coupled Linac.)

The Cyclinac solution is ideal for achieving an efficient distribution of active dose exploiting the special structure and temporal intensity achievable. Indeed spots beam can be produced at rate of $400 \mathrm{~Hz}$, allowing to adjust the intensity and position of ions in less than $2 \mathrm{msec}$, over an area of $20 \mathrm{~cm}^{2}$ both the traverse plan and deeply.

Another new frontier in machines for hadrontherapy is represented by FFAG (Fixed Alternate Gradient Field). Thanks to their fixed magnetic field and radio frequency modulation, 
the FFAG are syncrocyclotrons able to produce an intense pulsed beam. Compared to these machines synchrotron allows a greater pulsing frequency and raised acceptability in a moment. As for the acceleration of protons and light ions, all designed FFAG are the "scaling" type. This means that the shape of the optical beam and the frequency of betatron are held constant and independent from energy, and the magnets are built with a constant index field. For the hadrontherapy in Chiba (Japan) is designed a FFAG with three rings with a diameter of the circumferences of 3:4:5. The two most inner rings are used to accelerate protons to $250 \mathrm{MeV}$. The whole machine is used to accelerate beams of carbon $400 \mathrm{AMeV}$ with an intensity of some $10^{9} \mathrm{pps}$ and a repetition frequency of $200 \mathrm{~Hz}$. The machine is injected by an RFQ and a Linac both for protons and carbon ions.

\section{REFERENCES}

[1] Text books: U. Amaldi, B. Larsson Eds., "Hadrontherapy in oncology," Elsevier Science, ISBN 0-444-81918-5, 1994;

[2] J. Alonso, "Review of ion beam therapy: present \& future," EPAC00, 2000;

[3] R. Stone et al, "Preliminary report on fast neutrons in the treatment of malignant disease," Radiology, 35, p322, 1940.

[4] R.R. Wilson, "Radiological use of fast protons," Radiobiology 47, p487, 1946.

[5] A. Lennox, "Hadronic radiotherapy," FNAL 94/204, 1994;

[6] Chen Wuzhong, "Design of a light ion medical synchrotron," GSI-92-24, ISSN 01714546, 1992;

[7] W. Chu et al, "Performance Specifications for a Proton Medical facility," LBL Report 33749, 1993.

[8] G. Coutrakon, "Proton Synchrotrons for Cancer Therapy," CAARI 16, AIP Conf. Proc. 576, p861, 2001.

[9] S. Peggs, "Fundamental Limits to Stereotactic Proton Therapy," Trans. Nucl. Sci. 51, 3; or BNL C-AD/AP/121, 2004.

[10] A. Lomax et al, "Treatment planning \& verification of PT using spot scanning," Med. Phys. 31 (11), p3150 2004;

[11] J. Flanz et al, NIMB 99 p830, 1995.

[12] P. Miller, "The MSU Superconducting Cyclotron Program," Proc. $10^{\text {th }}$ Int. Conf. on Cyclos. p11, 1984;

[13] M. Schippers et al, "Beam-dynamics studies in a $250 \mathrm{MeV}$ superconducting cyclotron,” Nukl. 48 Supp2 p145, 2003;

[14] A. Geisler et al, "Status Report of the ACCEL $250 \mathrm{MeV}$ Medical Cyclotron," Cyclotrons04, p178, 2004.

[15] H.Blosser et al, IEEE Trans. Magnetics 25 125, 1989; 
[16] J. Parrell et al, "Advances in Nb3Sn strand for fusion \& particle accelerators," Trans. Appl. Superc., 15 p1200, 2005;

[17] G. Coutrakon et al., "Beam Optics for Scanned Proton Beam at LLUMC," CAARI 17, AIP Conf. Proc. 680, p1116, 2003;

[18] K. Umegaki et al, 'Hitachi Review Vol. 52 No. 4 p196-201, 2003.

[19] L. Badano et al, "Synchrotrons for hadron therapy: Part I," NIM A, 430, p512, 1999;

[20] News, "Antiprotons could help fight against cancer," p5; Amaldi \& Kraft, "Particle Accs. fight against cancer," p17; P-R. Kettle, "COMET brings promising future to PT," p24; CERN Courier Vol. 46 No. 10, December 2006.

[21] K. Noda et al, "Slow beam extraction by a transverse RF field with AM and FM," NIM A, 374, p269, 1996;

[22] N. AlHarbi et al, "Design of a compact synchrotron for medical applications," Rev. of Sci. Instruments, 74, p2540, 2003;

[23] S. Peggs (Ed.), "Pre-Conceptual Design of a Rapid Cycling Medical Synchrotron," BNL C-A/AP/6, 1999;

[24] R. Becker, "Modern Options for Hadron Therapy of Tumors," Rev. Sci. Instrum. 77, 2006.

[25] D. Trbojevic et al, "SC Non-Scaling FFAG Gantry"; PAC07, 2007

J. Pasternak, "Spiral FFAG for Protontherapy"; PAC07, 2007

[26] G. Caporaso et al, "High Gradient Induction Accelerator”; PAC07, 2007

[27] S. Akulinichev, "Radiological Centre at INR RAS"; PAC07, 2007

M. Maggiore et al, “300 AMeV SC Cyclotron”; PAC07, 2007

[28] M. Craddock, "The rebirth of the FFAG.” CERN Courier, Vol. 44, No. 6, 2006.

[29] D. Tirbojevic et al, "Carbon/proton therapy: A novel gantry design," PRST 10, 5, May 2007;

[30] A. Lennox \& R. Hamm, "A Compact Proton Linac for Fast Neutron Cancer Therapy," Proc. $3^{\text {rd }}$ Intnl. Topical Meeting on Nucl. Apps. of Accelerator Technology, ANS, p33, 1999;

[31] News, "LLNL, UC Davis team up to fight cancer," 2006,

http://www.llnl.gov/pao/news/sciencefeatures/2006/SF-06-04-02.html;

[32] J. Flanz, "Large Medical Gantries,” PAC95, 1995;

[33] M. Pavlovic, "Beam optics study of the gantry system for light-ion cancer therapy," NIM A, A399, p439, 1997; 